\title{
EL DOMINIO COMPARTIDO DE LA INVESTIGACIÓN Y EL DESARROLLO PROFESIONAL. UNA EXPERIENCIA EN MATEMÁTICAS CON MAESTRAS
}

\author{
Climent, Nuria y Carrillo, José \\ Didáctica de la Matemática \\ Universidad de Huelva
}

\begin{abstract}
Resumen. Este artículo es fruto de un proyecto de investigación entre dos investigadores y tres maestras en el que se vincula la investigación educativa con la formación del profesor.

Parte de una concepción de la formación del profesor como un proceso que sólo administrativamente sufre una separación entre formación inicial y permanente. Presta atención al conocimiento profesional de las maestras, considerando sus componentes, entre las que se incluyen las concepciones, actitudes y capacidades de dichas maestras sobre la matemática y su enseñanza. Ofrece un modelo de desarrollo profesional en el que se enfatiza el papel de la reflexión, aportando datos que ayudan a concretar este papel. Asimismo, se precisa cómo se ha proyectado el concepto de investigación colaborativa en nuestro trabajo y se dan sugerencias para la formación (inicial y permanente) de maestros y la investigación sobre dicha formación, todo ello dentro del ámbito de la educación matemática. De modo particular, se describe el contenido de algunas de las sesiones del proyecto, inspiradas en el análisis de situaciones de aula de las propias maestras y en situaciones de resolución de problemas matemáticos.

Palabras clave. Desarrollo profesional, investigación colaborativa, conocimiento profesional, formación de maestros, enseñanza de la matemática.
\end{abstract}

Summary. This paper derives from a research project carried out by two researchers and three primary teachers, linking educational research with teacher training.

We assume that teacher training is a process that only administratively is divided into pre-service and in-service training. Our perspective takes into account the primary teachers' professional knowledge, considering its components, in particular their beliefs, attitudes and capacities about mathematics and its teaching. It contributes a model of professional development in which we emphasise the role of reflection, giving some data that help concrete such role. Moreover, we clarify how the concept of collaborative research has been applied in our work; we also suggest some ideas about (pre- and in-) service teacher education and about research (in the realm of mathematics education). In particular, one describes the content of some sessions of the project, based on the analysis of teaching situations (of the primary teachers themselves) and on the tackling of mathematical problem solving.

Keywords. Professional development, collaborative research, professional knowledge, teacher training, mathematics teaching.

\section{INTRODUCCIÓN}

Resulta difícil atender de forma equilibrada los dos frentes principales que tiene ante sí cualquier profesor universitario de didáctica de la matemática: la docencia a futuros profesores y la investigación. Es bastante frecuente que se menosprecie la primera, valorando casi exclusivamente la investigación, probablemente por poseer criterios más claros y objetivos. En este artículo reivindicamos la importancia de la docencia universitaria en este campo y subrayamos la necesidad de que algunas investigaciones localicen sus objetos de estudio y sus conclusiones en la relación entre dicha docencia y la investigación.

No debe confundirse esta relación con la existente entre teoría y práctica, pues tanto la docencia como la investigación poseen su teoría y su práctica. De esta forma, las frecuentes asociaciones teoría-investigación y prácticadocencia son inexactas, aunque no carecen de sentido ${ }^{1}$. A lo largo de los siguientes apartados confiamos en poner de relieve que lo que aquí presentamos supera tales 
asociaciones, nutriéndose del flujo de relaciones que se producen en los ricos terrenos intermedios.

Uno de nuestros principales pilares es la formación del profesor. De hecho, esta investigación se ha llevado a cabo dentro del proyecto de investigación Desarrollo profesional a través de la investigación colaborativa sobre resolución de problemas, subvencionado por la Consejería de Educación y Ciencia de la Junta de Andalucía. Dicho proyecto está inmerso en la convocatoria de proyectos de formación permanente. En este proyecto de investigación han participado tres maestras de primaria y los dos autores de este artículo.

El trabajo que presentamos se sitúa en la práctica de una investigación en formación permanente. Del lado de los investigadores, esta práctica está respaldada, obviamente, por una teoría, a la que haremos mención posteriormente. Del lado de las maestras, su práctica docente es el punto de partida de las reflexiones sobre su conocimiento profesional. Tratarán de expresar sus opiniones sobre la docencia en primaria, y todos intentaremos obtener características deseables del conocimiento profesional del maestro de primaria en matemáticas. Esto nos permitirá establecer algunas conclusiones sobre modelos formativos de maestros. De esta forma, lo que inicialmente es una investigación en formación permanente se vincula con la docencia en formación inicial, a través de una red de teorías y prácticas (en lo que antes hemos llamado terrenos intermedios).

\section{MARCO TEÓRICO}

Ya hemos dicho que uno de nuestros principales pilares es la formación del profesor:

«Entendemos formación del profesor como un proceso interactivo (inmerso en un contexto social, organizativo, cultural...), básicamente entre formadores y estudiantes, pero incluyendo también las interacciones sistemáticas entre profesores dirigidas al crecimiento profesional. Al mismo tiempo, podemos ver la formación del profesor como un entorno de aprendizaje para todos los involucrados en este proceso de interacción.» (Krainer y Goffree, 1999, p. 295)

Además, la formación del profesor debería ser entendida como un proceso en el que la separación entre la formación inicial y la permanente es, fundamentalmente, administrativa (Carrillo y Coriat, 1999). Temas y características de la formación inicial debieran formar parte de la formación permanente, y viceversa.

Otro pilar fundamental es el conocimiento profesional, en el que consideramos diferentes componentes, tomando como referentes, entre otros, los trabajos de Bromme (1994) y Carrillo, Coriat y Oliveira (1999), ambos coincidentes en lo esencial respecto a las principales fuentes: pedagógica, psicológica, sociológica y epistemológica. En el conocimiento del contenido matemático (relacionado con la fuente epistemológica), distinguimos el conocimiento de y sobre matemáticas (Ball, 1990a); el primero, referido al conocimiento de hechos, procedimientos, conceptos, las relaciones entre éstos, y los significados y principios subyacentes; y el segundo, referido a la comprensión de la naturaleza del conocimiento matemático y de la matemática como cuerpo de conocimiento. Como conocimiento integrador de las cuatro fuentes, consideramos el conocimiento didáctico del contenido, o conocimiento de la materia para su enseñanza, que incluye el conocimiento de los modos de representación más adecuados para facilitar su comprensión y el conocimiento de las características del aprendizaje de los contenidos (Shulman, 1986; Blanco, Mellado y Ruiz, 1995). Incluimos en el conocimiento didáctico del contenido el conocimiento de materiales curriculares para la enseñanza de los contenidos (componente diferente del conocimiento de didáctico del contenido para Shulman, 1986). Por nuestra parte, hemos diferenciado, de los anteriores, el conocimiento de los currículos oficiales relativos a la enseñanza de la materia. El conocimiento pedagógico general, componente del conocimiento profesional del profesor para la enseñanza de las matemáticas, no ha sido objeto de nuestro estudio. Aunque de diferente naturaleza que los componentes descritos, consideramos también, dentro del conocimiento profesional del profesor, sus concepciones, actitudes y capacidades en relación con la materia y su enseñanza ${ }^{2}$.

Al objetivo de ampliar el conocimiento científico se une el de favorecer el desarrollo profesional de las maestras que intervienen en el proyecto. Este desarrollo profesional no es un simple modernismo respecto al término cambio profesional. Mientras éste encierra una actitud de superioridad del investigador respecto del profesor, aquél da a entender que lo más importante es propiciar espacios de reflexión sobre el propio conocimiento y las propias creencias, siendo esto el eventual punto de partida de un cambio elegido por el profesor. Por su parte, la perspectiva, analítico-positivista da paso a la humanista al hablar de desarrollo profesional. En esta perspectiva los profesores se expresan en sus propios términos, no en los de los investigadores, quienes tratan de comprender a aquéllos desde su propia visión.

Coincidimos con Imbernón (2000) en su propuesta de procesos de formación que desarrollen «instrumentos intelectuales para facilitar la reflexión sobre la práctica docente, y cuya meta principal sea aprender a interpretar, comprender y reflexionar sobre la enseñanza [superando] el concepto de formación como el dominio técnico de las disciplinas y [estableciendo] nuevos modelos relacionales, lo que significa diseñar una formación más descriptiva, no normativa» (p. 84).

Es objetivo de este artículo ofrecer un modelo de desarrollo profesional, al mismo tiempo que un medio de indagar en algunos aspectos del conocimiento profesional de los maestros. Detallaremos el papel de la reflexión en el proceso de construcción de conocimiento profesional (desarrollo profesional), siguiendo a Cooney y Krainer (1996), quienes enfatizan «un componente reflexivo de los programas de formación permanente en el que los 
profesores consideren explícitamente las implicaciones de sus propias experiencias de aprendizaje para su enseñanza» (p. 1162).

Procuramos aportar datos que saquen a la reflexión de la ambigüedad en la que suele situarse. Asimismo, trataremos de precisar la versión real que en nuestra investigación ha adquirido la colaboración (investigación colaborativa) inicialmente planteada. Finalmente, haremos algunas sugerencias para la formación inicial de maestros.

\section{EL ESTUDIO}

El interés inicial de la investigación ha partido de las maestras. Su preocupación por la mejora de su práctica les ha llevado a buscar la colaboración de los formadores para emprender un camino, no de formación externa proporcionada por estos últimos, sino de investigación en y sobre su propia práctica para confrontarla con otros modos de enseñanza de la materia. En otras palabras, querían crear un entorno de aprendizaje profesional, una subcomunidad de práctica, en la que desarrollar las cuatro dimensiones que, de acuerdo con Krainer (1998), caracterizan la práctica profesional: acción, reflexión, autonomía y comunicación. De hecho, como sucede habitualmente, trataron de desarrollar, sobre todo, reflexión, y también comunicación. Querían ampliar el entorno en el que construyen su conocimiento profesional: la interdependencia entre los dos sistemas autónomos que modelizan la enseñanza y el aprendizaje de las matemáticas (los procesos de aprendizaje de los estudiantes y el proceso interactivo de enseñanza) (Steinbring, 1998).

Por su parte, a los investigadores también les interesaba colaborar con las maestras, dentro de su programa de investigación sobre el conocimiento profesional.

El diseño previo del plan de actuación fue realizado de manera conjunta por todos los miembros del equipo mediante el consenso respecto a los diversos intereses de los participantes y la vía de alcanzarlos en un proyecto común. Se decidió centrar la atención en las concepciones de las maestras sobre la enseñanza y el aprendizaje de la matemática, y en su actuación en el aula. El proyecto comenzaría con el estudio de la situación de partida de cada maestra y su contraste con lo que considerábamos deseable (que determinaríamos mediante lecturas y discusiones), para, con este contraste y analizando los problemas de la práctica de cada una, extraer características deseables del conocimiento profesional de los maestros de primaria respecto a la enseñanza de las matemáticas y diseñar un plan que permitiera a las maestras modificar su actuación en el aula. Éste consistiría en la preparación, justificación y fundamentación conjunta de una secuencia de enseñanza, su puesta en práctica por parte de las maestras y el análisis de éstas conforme al perfil deseable. Como resultado de todo este proceso esperábamos contribuir al desarrollo teórico y práctico de una propuesta de conocimiento profesional deseable del maestro de primaria y el papel de la resolución de problemas en este conocimiento, iniciar en las maestras un proceso de desarrollo profesional y extraer consecuencias para potenciar el desarrollo profesional de los maestros en su formación permanente. Asimismo, por parte de los investigadores se pretendía obtener ideas que contribuyeran a mejorar los programas de la formación inicial. Sin embargo, en el transcurso del trabajo, han surgido varios aspectos inesperados que han hecho modificar el diseño y ampliar el foco de interés.

Por un lado, ha existido una desigual implicación de las maestras en el proyecto, diferenciándose en el esfuerzo invertido; asimismo, el grado de convencimiento de que su desarrollo profesional vendría dado por su reflexión y autoanálisis de su práctica ha diferido en las tres participantes. Hay que señalar que la lejanía a estos presupuestos ha sido directamente proporcional a la cercanía de sus concepciones sobre la enseñanza y el aprendizaje de la materia a una concepción tradicional. De este modo, se ha destacado el papel de una de las maestras, Ana, de concepciones investigativas, en la que el análisis y la discusión sobre sus concepciones y su práctica fue desvelando que su desarrollo profesional pasaba por convertir sus concepciones verbalizadas en concepciones en la acción. Así, esta maestra ha entrado en un proceso perso$\mathrm{nal}^{3}$ de cambio de su práctica docente, intentando acercarla a sus concepciones, y este proceso ha ido aportando información al grupo sobre su conocimiento profesional a la vez que ha servido para extraer información sobre el conocimiento profesional de las otras dos maestras. Por otro lado, y en relación con lo anterior, en el análisis de la actuación de Ana, los investigadores han observado que los aspectos más «débiles» de su conocimiento profesional residen en su conocimiento de y sobre matemáticas, $\mathrm{y}$, en menor medida, en su conocimiento didáctico del contenido.

Esto ha llevado a ampliar el foco inicial de estudio de las concepciones de las maestras y su actuación a su conocimiento de y sobre matemáticas y a su conocimiento didáctico del contenido.

Además, se ha considerado relevante que las propias maestras expliciten la importancia que atribuyen a los aspectos antes mencionados de su conocimiento profesional. Con este objetivo, se ha indagado sobre cuál es, desde su punto de vista, el conocimiento profesional deseable del maestro de primaria para la enseñanza de las matemáticas.

Las participantes en este estudio son tres maestras de educación primaria, con una experiencia de entre 12 y 25 años, una de ellas especialista en niños con necesidades educativas especiales. Este último hecho no ha diferenciado la participación de esta maestra en el proyecto. Su interés era el mismo que el de las maestras restantes (su desarrollo profesional), la única diferencia es que su práctica difiere de la de las otras dos maestras. Además de las características que le imprime el hecho de ocuparse de niños con dificultades especiales, suele trabajar con grupos pequeños, a veces de un solo alumno, y su misión suele ser reforzar en estos niños la labor que hace el maestro del aula correspondiente. Su autonomía en esta tarea es, por tanto, relativa, ya que tiene que seguir las pautas dictadas por el maestro del aula en muchas ocasiones. 


\section{METODOLOGÍA}

El planteamiento inicial del proyecto era el de una investigación colaborativa en la que todos los miembros del grupo actuaran como investigadores y algunos de ellos (las maestras) además como informantes ${ }^{4}$. Esta distribución de roles se ha respetado en lo referente al tratamiento de la información obtenida en relación con las concepciones de las maestras sobre la enseñanza y el aprendizaje de las matemáticas. No obstante, en otros casos, sobre todo en lo referente a la información sobre el conocimiento didáctico del contenido y el conocimiento de y sobre matemáticas de las maestras, el papel de investigadores ha sido realizado por los formadores, y las maestras han jugado sólo el papel inicial de informantes. Posteriormente, los resultados del análisis realizado por los formadores han sido discutidos en el grupo, por lo que las maestras han participado en esta discusión.

Es decir, las maestras han sido investigadoras en lo relativo a sus concepciones y no en lo que respecta a su conocimiento de contenido matemático (donde incluimos el de y sobre matemáticas) ni a su conocimiento didáctico del contenido. En el primer caso, la discusión de documentos sobre la enseñanza y aprendizaje de las matemáticas (principalmente sobre el papel de la resolución de problemas) y de investigaciones en concepciones de los profesores permitió que adquirieran una formación como investigadoras que les posibilitó actuar como tales, conjuntamente con los formadores. Aun manteniéndose en algunas maestras ideas bastante «tradicionales» respecto a la enseñanza y el aprendizaje de la materia, la familiarización con el instrumento de análisis (Carrillo, 1999) y el clima de reflexión y autocrítica que se ha propiciado en las sesiones de trabajo han sido suficientes para llevar a cabo el análisis de sus concepciones con ellas mismas participando como investigadoras. Sin embargo, en el caso del estudio de su conocimiento de contenido matemático y su conocimiento didáctico del contenido, resultó inviable desarrollar un proceso adecuado de formación a medio plazo que permitiera extraer la información necesaria. Tal proceso habría supuesto un desarrollo profesional de las maestras en lo referente a estos aspectos de su conocimiento profesional. Simon (1994), al referirse a los learning cycles, describe relaciones entre el aprendizaje de matemáticas por parte de los profesores, su aprendizaje sobre el aprendizaje de las matemáticas y su aprendizaje acerca de la enseñanza de las matemáticas, resaltando el carácter recursivo de la relación entre aprender matemáticas y aprender a enseñar matemáticas.

No obstante, las maestras del estudio no compartían la necesidad de reflexionar y profundizar en su conocimiento matemático, por lo que en el diseño inicial conjunto no incluimos su aprendizaje de matemáticas, aunque posteriormente, como se verá, se abordó a través de la resolución de determinados problemas.

En definitiva, las maestras aportan información, que se analiza en el grupo o sólo por los formadores. En cualquier caso, los resultados del análisis son discutidos en el grupo.

Detallemos brevemente cómo se ha obtenido la información y qué tratamiento se le ha dado (expuesto sintéticamente en las tablas I, II, III, IV y V).
Siguiendo el diseño previo, en los primeros meses del proyecto hemos abordado la discusión en grupo sobre distintos aspectos de la enseñanza y el aprendizaje de la matemática en primaria y sobre el papel de la resolución de problemas en dicho proceso. En estas discusiones hemos incluido el debate sobre artículos y documentos de investigación en los campos señalados. Los miembros del grupo hemos ido tomando notas de estas sesiones. Este trabajo nos ha permitido, por un lado, comenzar a explicitar y a indagar sobre las concepciones de cada maestra y, por otro lado, crear un lenguaje y un marco de referencia comunes al grupo, y familiarizarnos con lo que iba a ser el instrumento de análisis de estas concepciones: el instrumento para el análisis de las concepciones del profesor sobre la enseñanza y el aprendizaje de la matemática (Carrillo, 1999) ${ }^{5}$.

Una vez introducidos en la investigación, nos hemos dispuesto a obtener información sobre la actuación de las maestras. Para este objetivo se ha grabado en vídeo una sesión de cada una de ellas y éstas han elaborado un diario de sus sesiones (siempre en lo relativo a la enseñanza de contenidos matemáticos). Las sesiones han sido grabadas por uno de los formadores, que ha presenciado la clase como observador no participante ${ }^{6}$. La cámara se ha situado en un lugar fijo de la clase, que permitiera recoger la actuación de cada maestra y su interacción con los niños. El foco de atención, coherentemente con el objetivo perseguido, ha sido la propia maestra; la actuación de los niños sólo ha interesado en la medida en que ha aportado información sobre la actuación de ésta. Con cada maestra hemos seguido el siguiente proceso:

- Una vez obtenida la grabación de una sesión de clase, la hemos analizado conjuntamente en una sesión del grupo. Este análisis se ha llevado a cabo sobre la base de un análisis previo de la grabación por parte de los formadores, dando cabida asimismo a los aspectos que los miembros del grupo iban destacando durante el visionado conjunto.

- Después hemos analizado su diario (primero individualmente y luego en común).

- Finalmente, contrastando ambos análisis y teniendo en cuenta lo explicitado por la maestra en las sesiones previas del proyecto, hemos elaborado, también primero de forma individual para después consensuarlo entre todos, el perfil de las concepciones de ésta respecto a la enseñanza y el aprendizaje de la matemática.

Es difícil en este proceso separar los momentos de recogida de información de los de análisis de la misma. En cualquier tarea de análisis se está recogiendo información en paralelo. Por ejemplo, al analizar el vídeo, cada maestra refleja sus propias concepciones, y las declaraciones correspondientes son anotadas, de modo que forman parte de la información recogida sobre sus concepciones verbalizadas. Ése es el sentido del análisis previo de las grabaciones por parte de los formadores, a fin de poder usar el visionado de éstos y su análisis para recoger información de las maestras. 
Tabla I

Recogida de información sobre las concepciones de las maestras, su tratamiento y datos que aporta.

\begin{tabular}{|l|l|l|}
\hline \multicolumn{1}{|c|}{ Recogida de información } & Tratamiento de la información & \multicolumn{1}{|c|}{ Resultados sobre... } \\
\hline $\begin{array}{l}\text { Grabación de sesiones de aula y diarios de } \\
\text { aula de maestras }\end{array}$ & $\begin{array}{l}\text { Discusión de grabaciones (grupo), análisis de } \\
\text { diarios (grupo) y discusión del perfil de cada } \\
\text { maestra respecto a CEAM }\end{array}$ & CEAM, CmatEscolar \\
\hline $\begin{array}{l}\text { Notas de campo de las discusiones en } \\
\text { grupo sobre matemáticas y su enseñanza- } \\
\text { aprendizaje y sobre las grabaciones de aula }\end{array}$ & $\begin{array}{l}\text { Sirven de argumentación para la discusión del } \\
\text { perfil de cada maestra }\end{array}$ & CEAM, CmatEscolar, CDC, Cde/sobreMat ${ }^{9}$ \\
\hline
\end{tabular}

Respecto al modo de obtención del perfil de las maestras queremos destacar dos aspectos. Uno de ellos es que nos reafirmamos en que, como se hizo en investigaciones anteriores (Contreras, Climent y Carrillo, 1999), el análisis individual por parte de cada investigador, seguido de la discusión conjunta, nos parece un modo de análisis que resta subjetividad al proceso y le aporta rigor, a la vez que solidez a los resultados. Por otro lado, el hecho de que la maestra protagonista del análisis sea uno de los miembros del equipo de investigación ha resultado ser una baza importantísima en este análisis. El grado de reflexión y el interés de la maestra en los resultados ha provocado que en diversas ocasiones haya sido la reflexión de ésta sobre su propio quehacer y pensamiento el que ha llevado a colocarla, en el análisis conjunto, en posiciones «menos deseables» de las que había sido catalogada por los otros miembros del grupo en el análisis individual.

Esto es lo que ocurrió a la hora de situar las concepciones de una de las maestras, Ana, sobre la participación del alumno en el diseño didáctico. Al estar diseñada la tabla de análisis de concepciones en función de cuatro tendencias, son cuatro los indicadores que explican los diferentes niveles de participación del alumno. Descartados los indicadores de las tendencias tradicional y tecnológica (el alumno no participa ni activa ni pasivamente en el diseño de las actividades, programa, etc.), en el análisis conjunto discutíamos si era más adecuado el indicador espontaneísta o el investigativo (el alumno participa indirectamente en el diseño didáctico a través de sus reacciones al trabajo del aula o el alumno participa directa o indirectamente en el diseño didáctico, respectivamente). Este último es considerado más deseable, teniendo en cuenta el «marco ideológico» sobre la enseñanza que habíamos ido construyendo en las sesiones previas del grupo, con las diversas discusiones y lecturas. Fue la propia argumentación de Ana la que nos hizo colocarla en el espontaneísta:

«En principio yo me puse en los dos (espontaneísta e investigativo) porque quizás no entiendo muy bien lo de la participación directa. De forma general, la considero indirecta [la participación de sus alumnos en el diseño].
A veces, de manera puntual, cuando algún alumno me ha hecho una pregunta, una propuesta en la que el chiquillo me plantea algo que era más adecuado que lo que yo pensaba... [Tras aclarársele la diferencia entre participación directa e indirecta del alumno en el diseño ${ }^{7}$ ] No, es indirecto, no se ponen a diseñar ellos.»

La tabla I resume el proceso de estudio relativo a las concepciones de las maestras:

Como ya explicamos, el proceso de puesta en práctica de las concepciones de Ana ha repercutido en el proyecto. Ana se sumergió en un proceso de investigación-acción sobre su práctica en el que ella misma recogía datos sobre su actuación (utilizando sobre todo un diario que completaba tras las sesiones), reflexionaba sobre ellos y sacaba sus conclusiones, las cuales hacían que modificara su práctica posterior. A los investigadores nos pareció interesante ampliar la observación de la actuación de esta maestra de una sesión (como se realizó en principio con las tres maestras) a una secuencia de enseñanza, con el objetivo de estudiar el proceso de desarrollo profesional y el conocimiento profesional de Ana «en acción». Ana fue haciendo patente las ventajas que supone que la propia maestra asuma el rol de conducir la investigación según sus intereses (Jaworski, 1998). En principio se trataba de dos proyectos, con intereses y métodos distintos, paralelos al proyecto común del grupo, en el que los interesados éramos la maestra protagonista y los formadores. Pero el planteamiento en el grupo de cuestiones surgidas en las grabaciones se vislumbró como un modo de recoger más información sobre el conocimiento profesional de las tres maestras (profundizando en aspectos apenas abordados hasta el momento, como el conocimiento didáctico y el conocimiento del contenido) y como una forma de promover su desarrollo profesional. De este modo, se convertía en cuestión de interés para todos los integrantes del proyecto; lo que iba a ser el estudio por parte de los investigadores del proceso de desarrollo profesional de Ana, ajeno al trabajo del proyecto de investigación, y el propio proceso de investigación-acción de Ana, quedaron ligados a él, convirtiéndose en proyectos diferenciados pero que se benefician mutuamente (como en una especie de simbiosis). 
La secuencia de enseñanza elegida ha sido la concerniente a polígonos y simetrías, que ha ocupado la mayor parte de la enseñanza de contenidos geométricos de Ana durante el pasado curso académico ${ }^{10}$. En total han sido grabadas diecinueve sesiones de clase. El modo de grabación, el papel del observador y el foco de atención se han mantenido respecto de las grabaciones antes mencionadas (puesto que el objetivo sigue siendo el mismo).

El investigador autor de las grabaciones ha ido tomando notas de los aspectos de conocimiento de contenido matemático y de conocimiento didáctico del contenido que ponía de manifiesto Ana en estas sesiones. Estas notas junto con el análisis posterior de los vídeos por parte de los dos investigadores han servido para el diseño por parte de éstos de cuestiones matemáticas y de conocimiento didáctico del contenido que han sido planteadas posteriormente a las tres maestras en sesiones de trabajo conjunto de los miembros del proyecto. Estas cuestiones han sido situaciones extraídas literalmente de las sesiones grabadas (que ponían de manifiesto problemas con el contenido matemático o con el contenido didáctico relativo) o inspiradas en alguno de los problemas surgidos en éstas. En otras ocasiones, la respuesta de las maestras a algunas de estas cuestiones ha proporcionado resultados sobre su conocimiento de contenido matemático o didáctico del contenido en los que los investigadores hemos profundizado planteándoles otros problemas (matemáticos o didácticos).

Un ejemplo de tales situaciones es el que sigue:

En una de las sesiones grabadas de Ana, los niños llegan a todas estas formas posibles de dividir un círculo ${ }^{11}$ en mitades:<smiles>c1ccc(C23CC4CC(CC(C4)C2)C3)cc1</smiles>
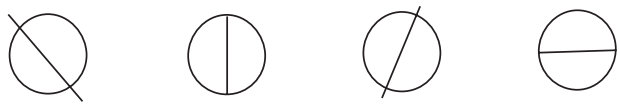

El primer caso se descarta, porque al doblar el círculo por los segmentos indicados por Ana, la parte doblada no cubre la central. En el segundo caso (que incluye el resto de las representaciones), los niños se dan cuenta de que es posible dibujar muchas de esas divisiones, pues se pueden dibujar «millones» de segmentos como los anteriores, sólo girando un poco un segmento (giro con centro en el centro de la circunferencia). Los niños no llaman a estos segmentos diámetros pero les imponen la condición de pasar por el centro de la circunferencia.

Uno de los niños aventura que el número de divisiones posibles dependerá del tamaño de la circunferencia, porque, si la circunferencia es mayor, girando poco a poco un segmento, podremos dibujar más segmentos que en el caso de la circunferencia menor («porque, si vamos dibujando los segmentos milímetro a milímetro, por ejemplo, en la circunferencia mayor habrá más [milímetros]». Ana decide no abordar esta conjetura en este momento, argumentando que «es otro asunto».

En una sesión del proyecto de investigación se plantea por qué se descartó el primer caso, si habría alguna posibilidad de que una partición de ese tipo produjera mitades y cómo se demuestra cada caso. Respecto de la segunda situación se les pide que se planteen la conjetura del niño sobre el número de posibilidades, dependiendo del tamaño de la circunferencia, y argumenten si es cierta o no.

Este tipo de cuestiones fueron planteadas por los investigadores a las maestras en sesiones del proyecto, provocándoles situaciones de conflicto que discutían entre ellas. El papel de los investigadores en estas discusiones era el de orientar la discusión y hacerles preguntas para que entraran en juego los distintos aspectos de su conocimiento profesional relacionados con la situación, así como recoger las intervenciones de las maestras (en notas de campo). Tras su intervención, los investigadores cambiaron su papel de orientadores de la discusión para participar en ésta, señalándoles los aspectos del conocimiento profesional que se habían puesto de manifiesto y discutiendo con ellas sus características.

Esta etapa ha tenido una doble vertiente: formativa y de investigación (no colaborativa). Ha sido de investigación para los formadores y para la maestra Ana, y formativa para todas las maestras. Queríamos tener el perfil de cada una de ellas para el diseño posterior del plan de actuación, tanto en lo referente a sus concepciones como a su conocimiento de contenido y didáctico del contenido. Al mismo tiempo, de igual modo que habíamos discutido en grupo las concepciones de las maestras y éstas se las estaban replanteando, pretendíamos que fueran conscientes de su conocimiento de contenido y didáctico del contenido y promover su desarrollo (mediante un proceso formativo). La discusión de las cuestiones planteadas ha cumplido este objetivo. Por su parte, nos hemos acercado al primero de ellos (obtener su perfil respecto a su conocimiento de contenido y didáctico del contenido) mediante el proceso de discusión conjunta antes explicado y a través también de los resultados del análisis que los investigadores han realizado de los datos recogidos en estas sesiones (esto, junto con el proceso de investigación-acción de Ana, conforman la vertiente de investigación de esta etapa del proceso).

Como se ha mencionado, el análisis por parte de los investigadores se ha realizado focalizando en aspectos del conocimiento de contenido y del conocimiento didáctico del contenido. Por ello, en la resolución de los problemas matemáticos propuestos, el objeto no ha sido analizar a las maestras como resolutoras sino cómo estas resoluciones ponían de manifiesto su conocimiento matemático (de $\mathrm{y}$ sobre). Por eso, no se ha realizado un análisis considerando fases de resolución o heurísticos empleados, y sí se han tenido en cuenta aspectos como el conocimiento del modo de argumentación y la prueba en matemáticas o el conocimiento de los contenidos implicados.

El resumen del proceso de estudio relativo al conocimiento de contenido y conocimiento didáctico del contenido de las maestras (diferenciando el proceso de investigación de los formadores y el formativo) se recoge en las tablas II y III: 
Tabla II

Proceso de investigación sobre el conocimiento de contenido matemático y el conocimiento didáctico del contenido de las maestras: recogida de información, su tratamiento y datos que aporta.

\begin{tabular}{|c|c|c|}
\hline Recogida de información & Tratamiento de la información & Resultados sobre... \\
\hline $\begin{array}{l}\text { Grabación en vídeo y observación por parte } \\
\text { de uno de los investigadores de una secuencia } \\
\text { de enseñanza de Ana }\end{array}$ & Análisis por parte de los investigadores & $\begin{array}{l}\text { CdeMat de Ana, CsobreMat de Ana, CDC } \\
\text { de Ana }\end{array}$ \\
\hline $\begin{array}{l}\text { Discusión en el grupo de situaciones de aula } \\
\text { extraídas de la observación de Ana }\end{array}$ & $\begin{array}{l}\text { Observación y análisis por parte de los in- } \\
\text { vestigadores }\end{array}$ & CdeMat $^{12}$, CsobreMat, CDC \\
\hline Resolución de problemas matemáticos & $\begin{array}{l}\text { Observación y análisis por parte de los in- } \\
\text { vestigadores }\end{array}$ & CdeMat, CsobreMat \\
\hline
\end{tabular}

Tabla III

Proceso formativo de las maestras centrado en su conocimiento de contenido matemático y su conocimiento didáctico del contenido.

\begin{tabular}{|c|c|c|c|}
\hline Fase 1a. & Fase 2a. & Fase 3a. & Resultados \\
\hline $\begin{array}{l}\text { Planteamiento por parte de los } \\
\text { formadores de situaciones de aula } \\
\text { y problemas matemáticos }\end{array}$ & $\begin{array}{l}\text { Discusión por parte de las maes- } \\
\text { tras }\end{array}$ & Discusión con los formadores & $\begin{array}{l}\text { Toma de conciencia y recons- } \\
\text { trucción del CdeMat, CsobreMat } \\
\text { y CDC }\end{array}$ \\
\hline
\end{tabular}

Finalmente, expliquemos cómo se ha llevado a cabo la parte del estudio concerniente a la recogida de información sobre el conocimiento profesional que las maestras consideran deseable para la enseñanza de la matemática. Al plantear a las maestras cuestiones relativas a su conocimiento de contenido y su conocimiento didáctico del contenido y discutirlas con ellas, hemos percibido que, mientras que son conscientes de las limitaciones que sus carencias en estos aspectos imponen a su desarrollo profesional, ambas no les preocupan del mismo modo. Aceptan como algo inevitable sus carencias de contenido y no se plantean la mejora de su conocimiento matemático como uno de los caminos de desarrollo de su conocimiento profesional. Esto nos llevó a pensar que es importante considerar las concepciones de las propias maestras sobre el conocimiento profesional que debe tener un maestro para la enseñanza de las matemáticas, ya que reflejará qué aspectos de su conocimiento profesional primarán en su desarrollo ${ }^{13}$. Al mismo tiempo, al reflexionar sobre esta cuestión y discutir sus distintos puntos de vista, se movilizan las concepciones de las maestras sobre la materia y su enseñanza y aprendizaje.

Para la recogida de esta información, en una de las sesiones del proyecto planteamos a las maestras la siguiente cuestión, haciendo que diferenciaran sus respuestas en dos columnas, la de la izquierda para plasmar lo deseable y la de la derecha para plasmar el estado de esas características (el objetivo de esta separación era que no confundieran lo deseable con sus perfiles):
«Explica qué características (en cuanto a conocimiento, actitudes, ideas, capacidades...) crees que debe poseer un maestro de primaria para enseñar bien matemáticas (escríbelas en la columna de la izquierda). ¿Cómo ordenarías en función de su importancia para la enseñanza las características descritas? (Enuméralas de más a menos importantes.)»

La respuesta de cada maestra a la cuestión anterior fue recogida y analizada por los investigadores conforme a una categorización previamente elaborada (sobre la base de los componentes descritos en el marco teórico). En esta categorización diferenciamos distintas parcelas que debían formar parte de este conocimiento profesional:

- conocimiento de matemáticas, conocimiento sobre matemáticas, conocimiento del currículo oficial de matemáticas, conocimiento didáctico del contenido y conocimiento pedagógico general;

- actitudes favorables hacia la matemática, su enseñanza, hacia el aprendizaje de los niños, hacia el trato humano con los niños y hacia sí mismo;

- concepciones adecuadas sobre la matemática escolar, sobre su enseñanza, sobre su aprendizaje, sobre «el mundo»;

- junto con ciertas capacidades (en la forma en que se han descrito en el marco teórico). 
Tras el análisis referido anteriormente, confeccionamos el guión para una entrevista conjunta en la que se pretendía que las maestras confrontaran sus puntos de vista argumentando sus posturas y saber si la ausencia de referencia a algunos aspectos de nuestra categorización se debía al olvido o a que no se consideraban aspectos importantes. Se llevó a cabo la entrevista-discusión de las características deseables señaladas por cada maestra y fue audiograbada. Los investigadores volvimos a analizar las ideas de cada maestra uniendo su respuesta a la cuestión inicial y lo que declararon en la entrevista.

Tras este análisis obtuvimos las características que cada maestra considera deseables (conocimiento profesional deseable, CPD, entendiendo el término conocimiento en un sentido amplio), clasificadas según nuestras categorías previas, y qué relevancia les atribuían.

Estos resultados fueron presentados a las maestras y discutidos con ellas.

En esta parte del estudio, al igual que ocurría en la anteriormente descrita, vuelve a verse la misma relación entre proceso de investigación realizado por los formadores y proceso de formación para las maestras. Los resultados de la investigación son de interés para promover el desarrollo de las maestras y el propio proceso seguido promueve este desarrollo.

Todo este proceso puede resumirse en las tablas IV y V.

\section{¿Una investigación colaborativa?}

Discutamos brevemente, considerando, entre otros, algunos de los aspectos señalados por Feldman, de qué manera se acerca nuestro estudio a una investigación colaborativa.

Este autor señala que «una investigación colaborativa "equitativa" es aquélla en la que los siguientes aspectos se aplican de igual manera para todos los "actores": trabajan a la par; asumen la misma responsabilidad en la identificación de los problemas, recogida de datos, análisis, y escritura de los resultados; comparten el mismo conjunto de objetivos; y se aseguran de que sus fines se alcanzan» (p. 343).

Teniendo en cuenta esta caracterización, pasamos a describir el posicionamiento de nuestro estudio:

a) El diseño inicial del proyecto es conjunto, participando todos los miembros del equipo e intentando que se ajuste a los intereses reales de todos.

Tabla IV

Proceso de investigación sobre el conocimiento profesional que las maestras consideran deseable para la enseñanza de la matemática en primaria: recogida de información, su tratamiento y datos que aporta.

\begin{tabular}{|l|c|c|}
\hline \multicolumn{1}{|c|}{ Recogida de información } & Tratamiento de la información & \multicolumn{1}{c|}{ Resultados sobre... } \\
\hline $\begin{array}{l}\text { Cuestión sobre el CPD (1). Entrevista poste- } \\
\text { rior a la cuestión (2) }\end{array}$ & $\begin{array}{l}\text { Análisis de 1 y 2 por los investigadores y dis- } \\
\text { cusión en grupo de los resultados del análisis }\end{array}$ & $\begin{array}{l}\text { Ideas de las maestras sobre CPD del maestro } \\
\text { de primaria para la enseñanza de la matemá- } \\
\text { tica }\end{array}$ \\
\hline
\end{tabular}

Tabla V

Proceso formativo de las maestras basado en la reflexión sobre el conocimiento profesional deseable para la enseñanza de la matemática en primaria.

\begin{tabular}{|l|l|l|l|}
\hline \multicolumn{1}{|c|}{ Fase 1a. } & \multicolumn{1}{|c|}{ Fase 2a. } & \multicolumn{1}{c|}{ Fase 3a. } & \multicolumn{1}{c|}{ Resultados } \\
\hline $\begin{array}{l}\text { Reflexión individual sobre el } \\
\text { CPD del maestro para la ense- } \\
\text { nanza de las matemáticas }\end{array}$ & Discusión entre las maestras & $\begin{array}{l}\text { Discusión de los resultados del } \\
\text { análisis realizado por los inves- } \\
\text { tigadores }\end{array}$ & $\begin{array}{l}\text { Replanteamiento de los aspectos } \\
\text { profesionales que consideramos } \\
\text { importantes. Reflexión sobre } \\
\text { el desarrollo profesional que } \\
\text { se quiere propiciar. Reflexión } \\
\text { sobre las concepciones sobre las } \\
\text { matemáticas y enseñanza y } \\
\text { aprendizaje. }\end{array}$ \\
\hline
\end{tabular}


b) Los objetivos de cada colectivo (maestras e investigadores) están diferenciados y son conocidos por todos. El objetivo de las maestras es su desarrollo profesional y el de los investigadores, la construcción de teoría. Tenemos un objetivo común (describir características deseables del conocimiento profesional de los maestros respecto a la enseñanza de las matemáticas), que, en el primer caso, es un medio para alcanzar el objetivo último y, en el segundo, tiene un carácter más finalista.

c) En la parte del estudio relativa a las concepciones de las maestras (Tabla I), la detección de los problemas, la recogida de datos, el análisis de los mismos y la extracción de resultados es llevada a cabo por todos los miembros del proyecto en un trabajo conjunto y consideramos que de manera «equitativa».

d) En la parte investigativa llevada a cabo por los formadores durante el resto del proceso (que engloba la recogida de las declaraciones de las maestras a lo largo de todo el proyecto, el análisis de la actuación de Ana y las partes del estudio concernientes al conocimiento de contenido y didáctico del contenido y conocimiento profesional deseable), las maestras han desempeñado el papel de informantes. La planificación, la recogida de datos, el análisis y la extracción de los resultados es realizada por los investigadores, por lo que esta parte no puede considerarse una investigación colaborativa.

e) Ahora bien, en esta etapa de la investigación, las maestras no se limitan a aportar la información. Este estudio les interesa, al profundizar en sus características profesionales, por lo que la recogida de información se realiza en las sesiones conjuntas del proyecto, consensuándose previamente que, aunque diseñadas por los investigadores, las tareas propuestas tienen sentido para ellas. Las maestras responden a estas tareas con la actitud especial de reflexión y confianza que les aporta el interés compartido. Además, los resultados del análisis son discutidos con ellas. Por otra parte, su interés en este estudio no es sólo en lo concerniente a los resultados del análisis correspondiente, el propio estudio es para ellas una vía de formación (además, para Ana, constituye una ampliación de su proceso de investigación sobre la acción, al posibilitarle el contraste de su propia reflexión sobre su práctica con la del resto de los integrantes del grupo). Queremos subrayar que el desarrollo profesional de las maestras no ha sido una consecuencia colateral de nuestro estudio, como ocurre en muchas de las investigaciones que se desarrollan teniendo a profesores como informantes (en su papel «tradicional»). Por el contrario, hemos diseñado el proceso para formar a los profesores, a la vez que llevar a cabo la recogida de la información para nuestra investigación. De no ser así, no se hubiera justificado la ligazón de esta investigación realizada por los investigadores con el proyecto común del grupo de investigadores y maestras.

f) La parte de investigación llevada a cabo por Ana es un proceso de investigación sobre su acción (Schön, 1992) en el que ella misma es la que realiza la detección de los problemas, la recogida de datos, el análisis de los mismos y la extracción de resultados. g) Los resultados de todas las investigaciones son compartidos por todos los miembros del equipo y discutidos por todos.

h) El proceso persigue en todo momento los objetivos de todos los participantes, discutiéndose previamente las tareas que se emprenden.

i) Consideramos que cada colectivo está satisfecho respecto a sus necesidades.

j) La publicación de los resultados de las investigaciones ${ }^{14}$ se realiza por parte de los investigadores. Esto es debido a que, en la discusión de los intereses de cada colectivo, las maestras pusieron de manifiesto no estar interesadas en tales publicaciones, por lo que no les compensaba invertir tiempo en esta tarea. Un asunto diferente es la publicación de materiales curriculares derivados del proyecto.

k) Hay cierta relación de dependencia entre ambos colectivos, pero no creemos que se pueda decir que hay una relación jerárquica, en el sentido de que un grupo ocupa un lugar más importante respecto del otro. Los investigadores son los «expertos» en las tareas de investigación y ello se pone de manifiesto constantemente en el proceso, pero las maestras tienen muy claro, y también se pone de manifiesto constantemente, que ellas son las «expertas» en la enseñanza.

Por todo lo anterior, pensamos que nuestro estudio tiene una parte colaborativa y una parte que, en cuanto a investigación, es no colaborativa, pero en la que la relación maestras-investigación es especial, al estar este proceso ligado a otro de investigación-formación en el que participan las maestras y al de investigación-acción de Ana.

\section{RESULTADOS}

No se han concluido los análisis de los tres procesos descritos pero ya tenemos algunos resultados que nos parecen interesantes desde el punto de vista de la investigación sobre el conocimiento profesional, así como desde el punto de vista del desarrollo profesional de las maestras.

\section{Desde el punto de vista de la investigación sobre el conocimiento profesional}

Presentaremos resultados de las concepciones de las maestras sobre la enseñanza y el aprendizaje de la matemática, su conocimiento del contenido y el conocimiento profesional que consideran deseable para la enseñanza de la materia.

No disponemos de resultados definitivos relativos a su conocimiento didáctico del contenido; sin embargo, el análisis realizado nos permite esbozar algunas pinceladas de este aspecto de su conocimiento profesional. Parece ser un conocimiento experiencial, fruto de su práctica docente, no fundamentado. Este hecho se obser- 
va tanto en la interpretación de las situaciones de aprendizaje que se producen en sus aulas como en el diseño e implementación de sus prácticas. Hay que diferenciar el caso de Ana, quien, por el proceso de investigaciónacción que está desarrollando y su mayor tendencia a la innovación, posee más conocimiento de materiales, recursos, modelos y estrategias para facilitar el aprendizaje. El conocimiento didáctico del contenido de Ana se diferencia del de sus compañeras no sólo en su contenido, sino también en su mayor grado de fundamentación, pero presenta limitaciones que originan problemas en su práctica docente. Estas limitaciones se derivan de abordar superficialmente ciertos aspectos del conocimiento didáctico del contenido en su autoformación, no afrontados como verdaderas situaciones problemáticas de su práctica.

El punto de vista de estas maestras hacia cualquier cuestión didáctica o de contenido es siempre el de la enseñanza. Esto es natural y deseable pero no si, como en estos casos, se busca la rentabilidad casi inmediata y eso impide analizar estas cuestiones desde otras perspectivas que repercuten en la enseñanza. Sin embargo, ellas no comparten la necesidad ni el gusto de abordar la resolución de problemas (matemáticos, sobre todo), aunque, como ya hemos explicado, la resolución de problemas como metodología en el aula de primaria es su principal tema de interés y la propugnan como beneficiosa. Este hecho es tan extremo que desde un principio las maestras del proyecto, y de forma especialmente tajante Ana, se negaron a que se trabajara la resolución de problemas matemáticos con ellas como resolutoras. Ha sido después de que ellas mismas hayan constatado sus dificultades en tales situaciones y su repercusión en su labor docente, cuando se han planteado en el proyecto estas situaciones.

\section{Concepciones de las maestras sobre la enseñanza y el aprendizaje de la matemática. El caso de Ana}

Dado que el caso de Ana ha sido el que mayor repercusión ha tenido en la marcha del estudio y que es sobre el que se ha obtenido más información por su especial implicación en el proyecto, detallaremos sólo el perfil de esta maestra. Los perfiles de las otras dos maestras, Sara y Lola, son de corte mayoritariamente tecnológico-tradicional, en el primer caso, y tecnológico-espontaneista con algunos indicadores de investigativo, en el segundo. En Lola (la maestra de educación especial) se manifiesta de manera muy fuerte una dualidad ideas-acción (más progresistas las primeras, más tradicional la segunda), mientras que en Sara se observa una práctica bastante tradicional, coherente con sus ideas.

El perfil de Ana, en lo relativo a sus concepciones sobre la enseñanza y el aprendizaje de la matemática, es mayoritariamente investigativo. Este perfil coincide en sus declaraciones y en su práctica, como se ha constatado en la observación de su proceso de investigación-acción. Ana diseña procesos investigativos para que los alumnos adquieran conocimientos conceptuales, procedimentales y actitudinales, otorgándole mucha importancia a los últimos.
El trabajo de los niños es de corte inductivo, estableciéndose conjeturas a partir de la observación y buscando su refutación o argumentación a favor. Se combina el trabajo individual con el trabajo en pequeños grupos o en gran grupo, dependiendo de la actividad a desarrollar. Ana considera que tanto las actitudes como las aptitudes de los alumnos pueden ser modificadas, pero es consciente de la dificultad de estas modificaciones en algunos casos. Opina que, «si pueden cambiarse es, desde luego, con una metodología basada en la resolución de problemas». Se pretende que el alumno sea consciente de su propio aprendizaje, para que le otorgue significado y aprenda verdaderamente; por eso la actividad del alumno incluye tiempo para la reflexión sobre lo que hace y por qué lo hace. En la clase se establece un ambiente dinámico, donde los alumnos, muy motivados por expresar sus ideas, comparten sus experiencias con el profesor y sus compañeros. El profesor actúa de guía y moderador en el debate de los alumnos, favoreciendo que respeten las opiniones de los compañeros y sean los propios niños los que argumenten a favor o refuten lo expresado por los otros, promoviendo una actitud crítica ante lo que se moviliza en el aula. Ana investiga en y sobre su acción. Parece que ideológicamente está a favor de la coordinación entre profesores, pero mantiene que en la práctica no siempre lo consigue (como le ocurrió en este curso), porque hay profesores con los que le resulta imposible coordinarse.

Ana mantiene ideas más tradicionales respecto a la participación de los alumnos en el diseño del proceso; éstos intervienen sólo de manera indirecta mediante sus reacciones. De igual modo, respecto a la consideración de los intereses de los alumnos como dinamizador del aprendizaje, considera necesario que los alumnos se interesen en lo que el maestro plantea y, con este objetivo, el maestro debe conseguir la motivación de sus alumnos, pero lo que interesa a los alumnos no es contemplado en el diseño de la secuencia de enseñanza-aprendizaje.

\section{El conocimiento de contenido matemático}

Aunque el estudio concerniente a esta cuestión no está finalizado, presentamos a continuación una aproximación a los resultados correspondientes.

En lo referente al conocimiento sobre matemáticas, las maestras de nuestro estudio no poseen apenas nociones de este conocimiento ni sabían siquiera de su existencia. En concreto, nunca se habían planteado el valor de los ejemplos y los contraejemplos en matemáticas, ni qué vías podían ser válidas para una demostración. Su modo de argumentación posee en muchas ocasiones errores similares a los de los alumnos de primaria o secundaria ${ }^{15}$. La vía de validación de una conjetura hecha por un alumno es conocer de antemano si es cierta o no. Si es una conjetura sobre la que no tienen conocimiento ni se puede deducir fácilmente de conocimientos que posean, no tienen herramientas (ni hábito) para enfrentarse a su prueba. Además, se encuentran con dificultades a la hora de construir una definición matemática. A menudo incluyen en una definición características que sobran, que se deducen de otras que ya están consideradas en la misma o dan definiciones demasiado «extensas», que incluyen ejemplos que no consideran dentro de ésta ${ }^{16}$. 
Su conocimiento de matemáticas se limita casi al de primaria, con un nivel de profundización no mucho mayor que el que adquieren los alumnos al término de esta etapa. Han olvidado y rehuyen los contenidos matemáticos «más avanzados» ${ }^{17}$. Esto hace que no tengan ninguna autonomía en lo que respecta a decisiones sobre el tratamiento del contenido ni el referente de la matemática como conjunto. Además, propicia la creación de conceptos erróneos en los niños. Su conocimiento matemático es además rígido y a imagen y semejanza del que se presenta en los libros de texto de la etapa; parece que hubieran adquirido este conocimiento durante su etapa docente y hubieran desechado, por no serles útil, el adquirido previamente. No establecen apenas relaciones entre los contenidos.

Quizás, como consecuencia de haber primado como docentes los contenidos aritméticos sobre los geométri$\cos ^{18}$, tienen mayores carencias en los segundos, aunque el conocimiento aritmético que poseen es mecánico y se basa fundamentalmente en el conocimiento de los algoritmos. Conocen la existencia de otros conjuntos de números, pero sólo saben desenvolverse con números naturales, enteros o decimales finitos (los que se trabajan en educación primaria), dándose situaciones sorprendentes con el tratamiento de otros números (como apreciaremos en el ejemplo que sigue).

En cuanto al poco conocimiento geométrico que poseen, es básicamente un conjunto de nombres, imágenes y definiciones muy rígidas y aprendidas de memoria.

Veamos algunas de estas carencias en la resolución, por parte de dos de las maestras (Ana y Sara), de un problema matemático que se les planteó en una de las sesiones del proyecto:

Se plantea a los alumnos una cuadrícula con el cuadrado exterior (de lado $8 \mathrm{u}$ ), cuyo lado queda dividido por el vértice del cuadrado interior en dos partes de medidas respectivas $2 \mathrm{u}$ y $6 \mathrm{u}$, siempre tomando como unidad de medida (u) la propia de la cuadrícula.

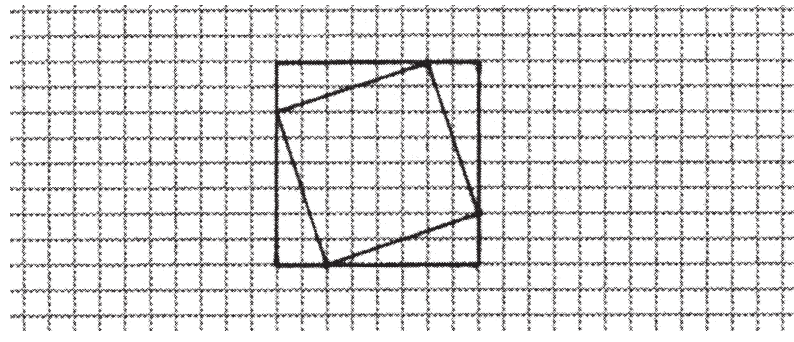

Formadores-investigadores: ¿Es el cuadrado interior la mitad (en el área) del cuadrado exterior?

Ana: ¡En una primera vista no lo sé! Creo que éstos dos [señalando los triángulos correspondientes a dos lados opuestos del cuadrado interior] son iguales. Y el rectángulo que se forma es la mitad del cuadrado [interior]. Y lo mismo con los otros dos... Tiene que ser el área de los cuatro triángulos la mitad de la del cuadrado grande. [Calcula el área del cuadrado grande y el de los dos rectángulos, en ambos casos contando las medidas de los lados por la cuadrícula y multiplicando los lados respectivos. No le sale la suma de las áreas de los dos rectángulos la mitad del área del cuadrado exterior]. No.
$F$ : ¿Cómo se puede inscribir un cuadrado en otro de manera que el exterior sea el doble del interior?

Ana: Tendría que tener área 32 . No hay ninguno, porque no hay ningún número que al cuadrado dé 32 .

$F: ¡ \sqrt{ } 32$ !

Ana: ¡ $\sqrt{ } 32$ ! ¡Para mí eso no!

$F$ : Números no enteros.

Ana: No, pero yo sólo miro números enteros.

$F$ : ¿Es un cuadrado lo de dentro? [Señalando el cuadrado interior del dibujo inicial]. Tiene área 40.

Ana: ¡No!

$F:$ ¿No es un cuadrado?

Ana: Parece que sí. [Lo justifica por los lados y los ángulos]

[Ana y Sara hacen el dibujo y comprueban que en este caso sí les sale la mitad]

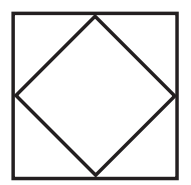

$F$ : El cuadrado del centro tiene área 32.

Ana: ¿Eso cómo puede ser si es un cuadrado?...Yo no sé hacer $\sqrt{32}$. [Se refiere a calcular una aproximación del número por el algoritmo de la raíz cuadrada. Sara efectúa el algoritmo]

$F$ : Así es por aproximación.

Sara: ¿Entonces cómo se puede poner de manera exacta?

$F:$ i $\sqrt{32}$ !

[Se les plantea que busquen más casos en que sea la mitad (como el anterior). Intentan hacerlo buscando que el lado mida 5 '... Se dan cuenta de que en la cuadrícula no pueden hacerlo (respetando que los vértices coincidan con los de la cuadrícula)].

[Sara dibuja la siguiente imagen]

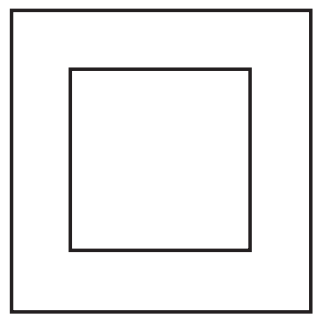

$F$ : Ése no está inscrito

Sara: No está inscrito, ¿por qué?

[Se les aclara lo que significa inscrito. Ana sigue intentando el caso inscrito]

Ana: No encuentro la manera de hacerlo respetando la cuadrícula.

$F$ : No tienes por qué hacerlo.

[A Ana no le salen cuadrados porque no coge las mismas medidas de los segmentos en que queda dividido cada lado del cuadrado exterior por los vértices del interior]

Ana: ¡Ya lo he hecho! [Ha dibujado casi la solución que encontraron antes. Al hacerle notar si es la misma que la anterior dice que no, pero se da cuenta de que tampoco es solución]

[Sara sigue intentando con los cuadrados no inscritos, sino centrados 
en el grande, con los lados paralelos a éste, como en el caso que antes se le aclaró que no correspondía a un cuadrado inscrito. Se le aclara que en ese caso nunca le va a salir por estar considerando lados enteros] [«Se dan por vencidas». No saben cómo encontrar otra solución, pero tampoco aventuran nada sobre si existirá o no, y, en caso afirmativo, cuántas más]

$F$ : ¿No se podía haber resuelto el problema de este modo? [Algebraicamente, haciendo uso de que los segmentos del lado del cuadrado grande que quedan determinados por el vértice correspondiente del cuadrado inscrito pueden considerarse como $x$ y $8-x$. De este modo se obtiene la ecuación

$$
4 \frac{x(8-x)}{2}=32
$$

Ana: Yo no sé resolverlo.

[Sara dice que sí sabe y llega a $16 x-2 x^{2}=32$ ].

Sara: ¡A ver si me acuerdo! ¡Si es hasta cuadrado! ¡Hay que acordarse de la fórmula del cuadrado!, raíz cuadrada de... [con la musiquilla típica de aprenderse algo de memoria].

[Sara llega a $8 x-x^{2}=16$; y de aquí a:

$$
\sqrt{8 x}-x=4
$$

Sara: ¡Esto no es así!, ¡espérate!, ¡para restar potencias...!, ¡no sé pasar!

$F:$ ¿Y si lo ponemos así: $x^{2}-8 x+16=0$ ?

[Sara tiene dificultades para ver cómo se ha llegado a eso. Ana parece que no. Se les recuerda la fórmula de resolución de las ecuaciones de segundo grado].

Sara: ¿Y eso no hay otra forma de hacerlo?...

En esta resolución se ponen de manifiesto sus dificultades con los números no naturales, y las restricciones que añaden al problema al imponer que las medidas de los lados del cuadrado interior deben ser números naturales. Incluso llegan a afirmar que no hay ningún número (que pueda ser medida de un lado) que al cuadrado dé 32. Esta misma idea les hace que duden de que el cuadrado interior con área $40 \mathrm{u}^{2}$ sea verdaderamente un cuadrado o que se sorprendan cuando consiguen dibujar un cuadrado con área $32 \mathrm{u}^{2}$. En su desconocimiento de los números irracionales, les sorprende también que, al intentar escribir $\sqrt{32}$ como un número decimal, el resultado sea siempre aproximado. El significado que tiene para ellas la raíz cuadrada de un número es el cálculo del algoritmo correspondiente. $\sqrt{ } 32$ como tal es inmanejable, se convierte en un escollo.

A pesar de ir buscando un cuadrado cuya área sea $32 \mathrm{u}^{2}$ y de haber obtenido uno con medida de los lados $\sqrt{32 u}$, cuando siguen buscando cuadrados con área, la mitad del cuadrado grande (es decir, de área $32 \mathrm{u}^{2}$ ), Ana lo hace intentando coger como lado del cuadrado 5'...u (con decimales finitos) y Sara con cuadrados centrados en el grande, esto es, con medida de lados naturales. Esto lleva a pensar que no se han planteado que el área del cuadrado determina unívocamente su lado o que, en el caso de Ana, se identifica $\sqrt{32}$ con un decimal finito del tipo 5'... En el caso de Sara, o no se da cuenta de que los cuadrados que dibuja siempre tendrán medida de lado natural, o no es consciente de la condición de que el área debe ser $32 \mathrm{u}^{2}$, o no le plantea problemas el hecho de que un número natural al cuadrado dé como resultado 32 .
La misma restricción de que la medida de los lados sean números naturales, a pesar de haber encontrado ya un cuadrado con lado $\sqrt{3} 2 \mathrm{u}$, les lleva a imponer que los vértices del cuadrado interior estén sobre la cuadrícula. No se hacen con el problema, pasándoseles por alto que, para dibujar un cuadrado inscrito sobre el dado, el vértice del cuadrado menor define sobre cada lado del cuadrado mayor dos segmentos de longitudes $x$ y 8 - $x$, con $x$ coincidente en cada lado.

Tienen serios problemas para resolver una ecuación de segundo grado y para operar y despejar identidades con raíces y exponentes.

Estas carencias son, desde nuestro punto de vista, de distintos tipos.

Por un lado, hay escollos (como no recordar lo que es un cuadrado inscrito o la fórmula de la ecuación de segundo grado) que pueden atribuirse al olvido, por ser contenidos que no se trabajan directamente en primaria. Pero el problema reside en que no se hace uso de estos contenidos porque las maestras no resuelven situaciones problemáticas que son presentadas con el contenido de primaria. Si los problemas y las situaciones que se plantea a los niños no son simple aplicación de reglas enseñadas previamente, y aun en estos casos, es natural que en la propia aula surjan situaciones o conjeturas que, si el maestro las aborda le llevarán inevitablemente a contenidos que no pertenecen a la etapa primaria pero que son básicos, como la ecuación de segundo grado o los números irracionales. Esto es lo que le ha ocurrido a Ana en su nuevo enfoque del aula ${ }^{19}$.

El caso de las dificultades con los números irracionales y la idea de que las medidas de los lados deben ser naturales nos parecen asuntos diferentes al anterior. En estos casos se poseen conceptos erróneos y visiones restringidas de los números no naturales y una visión casi «ingenua» de los números en la realidad. No sólo no tienen idea del «horizonte» de los contenidos que enseñan (en este caso los naturales) sino que la visión de los mismos contenidos que enseñan es irreal.

Finalmente, el hecho de que no se hagan con el problema, ni se planteen ninguna estrategia para abordarlo, es fruto de su escasa reflexión sobre la situación planteada. Parecen actuar «sobre la marcha», sin pararse a observar detenidamente la situación para aprehender las claves de ésta. Este hecho no es de extrañar si se considera (según sus propias declaraciones) que raramente abordan problemas matemáticos. La verdad es que nunca han hecho matemáticas (ni, como veremos después, se sienten atraídas por ello).

\section{Importancia que atribuyen a distintos aspectos de su co- nocimiento profesional}

Desde el punto de vista de Ana, lo más importante en las características profesionales de un maestro de primaria, pensando en la enseñanza de las matemáticas, es poseer inquietudes profesionales y personales y la idea de que 
nada está «acabado» (todo puede mejorarse y cambiarse), que le lleven a seguir aprendiendo. Además, es muy importante que posea una buena formación didáctica, seguida de formación matemática y, finalmente, una serie de actitudes positivas hacia los niños y hacia sí mismo, junto con una concepción de la enseñanza de la matemática cercana a la resolución de problemas.

Por su parte, Lola concede mucha más importancia al conocimiento matemático del maestro, considerando que es lo más importante en su conocimiento profesional, seguido del conocimiento didáctico del contenido (en lo que se refiere a estrategias metodológicas) y, por último, de unas actitudes adecuadas sobre todo hacia los niños y su aprendizaje.

Estos puntos de vista son coherentes con las cualidades profesionales de ambas maestras. Ana reflexiona más sobre su labor profesional que Lola, tiene concepciones más «cercanas a la resolución de problemas», como expusimos en el apartado correspondiente, y declara poseer menos conocimiento matemático del que declara poseer Lola ${ }^{20}$. Por su parte, Lola, puede que influida por su formación y su labor como especialista en niños con dificultades especiales de aprendizaje, se preocupa más por los problemas de aprendizaje y tiene más confianza en que muchos de estos problemas puedan subsanarse con, usando sus propias palabras, «una buena metodología» (se refiere a que el maestro ponga en juego en el aula estrategias metodológicas adecuadas).

El resultado relativo a la importancia que ambas atribuyen al conocimiento matemático es interesante. Mientras que Lola parece poseer mejor conocimiento de contenido, achaca las limitaciones de su práctica y los problemas que en ella se encuentra principalmente a su falta de conocimiento matemático y, consecuentemente, su propuesta de desarrollo profesional pasa por abordar su reconstrucción; por su parte, Ana declara saber menos de matemáticas y se constata, en su análisis (del que ella comparte los resultados), que los principales escollos de su práctica corresponden a carencias de conocimiento de contenido. Por ello, su principal vía de desarrollo profesional debería ser la profundización en este conocimiento. Sin embargo, coherentemente con el lugar en que Ana lo ha colocado en el «ranking» de importancia dentro de las características profesionales consideradas, no está dispuesta a enfrentarse a esta profundización. Sus declaraciones son claras:

«Yo no tengo ninguna curiosidad [respecto del estudio del contenido matemático]. Mi única curiosidad es enseñar mejor a los niños de lo que me enseñaron a mí, pero me he encontrado en el camino mi falta de conocimiento matemático. Si no tengo tiempo, ¿cómo lo voy a sacar de donde no tengo? Si me gustara, a lo mejor le sacaría tiempo. No tengo experiencia como estudiante de haber hecho eso [«hacer matemáticas»]».

Estas consideraciones relativas a lo que conceden importancia y a lo que se encuentran dispuestas a dedicar sus esfuerzos ha condicionado los objetos de estudio (en este proceso formativo de investigación), determinando su proceso de desarrollo profesional. No obstante, su progreso a lo largo del proyecto respecto a una más profunda toma de conciencia de sus necesidades como maestras ha llevado a abordar algunos de estos objetos (como, por ejemplo, su conocimiento de y sobre matemáticas).

\section{Desde el punto de vista del desarrollo profesional de la/s maestra/s}

Estos resultados son de distinta naturaleza que los del apartado anterior (no sólo por las distintas perspectivas desde las que los hemos agrupado, sino porque los primeros pretenden contribuir a la construcción de teoría, mientras que los segundos no persiguen esa construcción). Los resultados de los que nos ocupamos ahora constatan la incidencia de la investigación sobre el desarrollo del conocimiento profesional de las maestras. Describiremos esta incidencia atendiendo a diversos aspectos de su conocimiento profesional.

La toma de conciencia de las maestras de sus concepciones ha sido patente en el propio proceso de análisis de su perfil según sus concepciones sobre la enseñanza y el aprendizaje de la matemática, como ya explicamos. Ha propiciado que estas concepciones entren en conflicto con las nuevas ideas que se han estado discutiendo, produciéndose de este modo un replanteamiento de éstas y, por tanto, su evolución (entendiéndose como tal que la maestra reflexiona sobre tales concepciones y las confronta con otras perspectivas, fundamentando sus nuevas concepciones, ya sean iguales a las de antes o distintas $)^{21}$. Aunque los cambios en su práctica docente han sido más patentes en Ana, en Lola también se ha observado una evolución hacia actuaciones menos tradicionales. Este cambio no parece haber sido provocado tanto por una modificación de las concepciones como por conocer y sentir confianza en otros modos de enseñar y aprender matemáticas. El caso de Ana ha sido especialmente claro (ya hemos comentado en diversas ocasiones su acercamiento de las concepciones en la acción a las concepciones verbalizadas).

Las maestras han tomado conciencia de su conocimiento profesional y sus necesidades formativas y de ampliación (en lo relativo al conocimiento de y sobre matemáticas y conocimiento didáctico del contenido). Esta toma de conciencia ha estado propiciada por varios aspectos: la confrontación de situaciones cuyo abordaje ha puesto de manifiesto aspectos de su conocimiento profesional y sus limitaciones, el análisis del abordaje de estas cuestiones, el análisis de su práctica y, sobre todo, la reflexión de las maestras. También en este punto tenemos que destacar que los logros en este terreno, como en la mayoría de lo alcanzado en este estudio, tanto en cuanto al desarrollo profesional de las maestras como en cuanto a resultados desde el punto de vista de la investigación en el conocimiento profesional, se deben principalmente a la profunda reflexión de al menos dos de las maestras de este proyecto. Aunque en lo que respecta a la ampliación de esos aspectos de su conocimiento profesional se han conseguido menores logros que en los otros señalados, entre otras cosas por el comentado rechazo de alguna 
de las maestras a enfrentarse a problemas matemáticos, éstas han abordado aspectos de contenido matemático y didáctico del contenido al enfrentarse a las situaciones matemáticas y didácticas que nos han servido para indagar sobre éstos y al comentar posteriormente estas situaciones y su actuación.

\section{CONCLUSIONES}

Una de las objeciones de Feldman a algunas investigaciones que se declaran colaborativas es la queja de los investigadores hacia el escaso cumplimiento de los profesores en las tareas de investigación. Este autor hace un llamamiento que compartimos, para que se considere el lugar que desempeña la investigación dentro de las ocupaciones e intereses profesionales y personales de un profesor (frente al lugar que desempeña en el caso de un investigador). El desarrollo profesional de los maestros no puede pasar exclusivamente por su dedicación altruista. Deben buscarse vías de formación que faciliten este proceso y se adecuen a la disponibilidad de éstos. Además, desde la administración educativa debe contemplarse esta formación como parte de su dedicación profesional. De no ser así, siempre estarán implicados profesionales especialmente ilusionados, pero que al cabo de un tiempo no podrán mantener su interés y dedicación. No ha sido nuestro propósito, por tanto, criticar la labor de las maestras participantes (y por extensión al resto del colectivo), sino llamar la atención a los responsables de la administración educativa y a los formadores de maestros.

El proceso aquí descrito ha resultado ser una vía para iniciar un desarrollo profesional en las maestras participantes que, estamos seguros, continuarán ellas de manera autónoma. El modo en que se propicia la reflexión de las maestras y la movilización de sus ideas sobre la materia, su enseñanza y aprendizaje, sus características y necesidades profesionales y, sobre todo, la propia conciencia y decisión sobre su proceso de desarrollo profesional avalan que las maestras que se han implicado en el proyecto lo hayan hecho en un proceso personal de desarrollo profesional.

Por todo esto, proponemos la aplicación de este proceso como modelo de formación de profesores en ejercicio. Consideramos que es interesante, en este sentido, el trabajo conjunto de pequeños grupos de profesores e investigadores-formadores en un proceso común de investigación-desarrollo profesional. Este modelo incluiría, por tanto, procesos de toma de conciencia de las propias concepciones, discusión sobre cuáles son las concepciones deseables para la enseñanza de la materia, discusión conjunta sobre la propia práctica tomando ésta como fuente de situaciones problemáticas (teniendo presente que es necesario hacer un uso constructivo para los profesores del análisis de dicha práctica, no llevándoles a situaciones que podrían resultarles frustrantes) y reflexión sobre el propio proceso de desarrollo profesional.

Los resultados de esta investigación nos sugieren consecuencias para la formación inicial de maestros. Por un lado, las carencias en el conocimiento profesional de estas maestras nos hacen pensar en el contenido de los programas de formación de maestros (tanto inicial como permanente). Numerosas investigaciones habían subrayado la necesidad de abordar algunos de estos aspectos en la formación de profesores para la enseñanza de las matemáticas (entre las que destacamos los trabajos de Ball y sus colaboradores sobre el conocimiento de contenido matemático. Los artículos de 1988, 1990a, 1990b, 1990c, 1996 son una muestra de éstos). Coincidimos en la necesidad de abordar en la formación de maestros los aspectos estudiados de su conocimiento profesional: conocimiento de contenido en su vertiente de y sobre, conocimiento didáctico del contenido y concepciones sobre la materia y su enseñanza. En particular, creemos que es necesario profundizar en la influencia de la experiencia en el conocimiento de y sobre matemáticas. Conjeturamos la existencia de parcelas del conocimiento individual que no se plasman en los habituales exámenes de la formación inicial, las cuales, sin embargo, se potencian a lo largo de la vida profesional, consolidando un conocimiento del contenido que tiene coincidencias con el probado en exámenes, pero que al mismo tiempo posee partes diferentes. Es decir, hay partes ocultas del conocimiento de contenido del estudiante para maestro que sólo se activan a partir de la experiencia profesional.

Además de reforzar los resultados de los trabajos mencionados, es nuestro propósito proseguir la investigación con el objetivo de poner de manifiesto las repercusiones de estas limitaciones del conocimiento profesional sobre la práctica docente y sobre el propio proceso de desarrollo profesional.

Por otro lado, el proceso de investigación seguido sobre el conocimiento de contenido y didáctico del contenido de las maestras nos sugiere aspectos metodológicos para la formación inicial de maestros. La discusión de problemas matemáticos y didácticos puede ser una vía de formación de los estudiantes para maestro tan rica como lo ha sido en el caso de las maestras de nuestro proyecto. Además, las situaciones problemáticas surgidas en el proyecto se convierten en material de trabajo para la formación inicial. De este modo, nuestra práctica docente (la formación inicial de maestros) se nutre de los resultados de la investigación que desarrollamos (ejemplificando la deseada vinculación entre ambos frentes de nuestra profesión).

Tanto en el campo de la investigación en didáctica de la matemática como en el de la didáctica general, pero inmerso en el dominio de la formación y el desarrollo del profesorado, nos parece interesante seguir profundizando en las características reales de la puesta en práctica de investigaciones colaborativas, pues somos conscientes de la multiplicidad de interpretaciones en el diseño y, sobre todo, de la multiplicidad de realidades que configuran el carácter colaborativo de la investigación. Los distintos intereses y necesidades surgidos a lo largo del proceso, al lado de factores menos controlables, desfiguran en la práctica el diseño establecido inicialmente, desfiguración que no posee carácter peyorativo alguno, más bien al contrario, supone la adaptación al grupo y una muestra de la deseada vinculación entre investigación teórica y práctica. 
El protagonismo del profesor en su proceso de desarrollo profesional conlleva ciertas limitaciones. Como destaca Ball (1996), «iniciarse en un terreno que va más allá de los propios horizontes actuales es difícil, si no imposible» (p. 502). Sin embargo, si el diseño del proceso de desarrollo profesional es externo, es más difícil contemplar las necesidades y preocupaciones de los profesores. Las limitaciones en el horizonte del conocimiento profesional de las maestras nos llevaron a adaptar el carácter colaborativo de la investigación en algunos aspectos (en lo concerniente al conocimiento de contenido y conocimiento didáctico del contenido se hizo presente el carácter formativo). En el diseño de este proceso formativo (externo a las maestras) hemos intentado respetar sus preocupaciones, lo que ha provocado, como ya hemos comentado, asumir por parte de los investigadores la imposibilidad de abordar en profundidad ciertos aspectos de su conocimiento profesional. Nos planteamos, llegados a este punto, la necesidad de continuar en la búsqueda de modelos prácticos de formación-investigación que equilibren las necesidades e inquietudes de profesores e investigadores. Hemos pretendido constatar la ingenuidad que supone creer que una investigación colaborativa pura pueda permitir el desarrollo profesional permanente y continuo de los profesores; nos parece que existen modelos intermedios, donde los roles de unos y otros deben ser por supuesto, no sólo respetados, sino potenciados. La potenciación del protagonismo del maestro es un buen punto de partida para asumir posteriormente su responsabilidad en su proceso de desarrollo profesional, ya sea a través de experiencias de formación en grupo o bien a través de su propia práctica o experiencias de formación individuales.

Finalmente, queremos enfatizar la especial dificultad encontrada al tratar de incidir en el desarrollo del conocimiento de contenido de las maestras. Conjeturamos que se trata de un hecho bastante extendido en el colectivo de los maestros. Uno de nuestros retos futuros es proponer vías de formación que lo contemplen.

\section{NOTAS}

${ }^{1}$ Puig (1998) propone interpretar las relaciones mencionadas con el par objeto de conocimiento - conocimiento elaborado sobre el objeto.

\begin{abstract}
${ }^{2}$ Estas capacidades se refieren a características generales del individuo, como capacidad para tomar decisiones y hacerse responsable de ellas, no relacionadas con los componentes del conocimiento profesional. Las capacidades relacionadas con estos componentes, acordes con Ball y McDiamird (1988), se hallan incluidas básicamente en su dimensión procedimental.
\end{abstract}

\begin{abstract}
${ }^{3}$ Aunque ha sido propiciado por el trabajo en el grupo, no se ha planteado desde éste; ha sido una decisión tomada y emprendida individualmente por la maestra.

${ }^{4}$ En Feldman (1993) puede encontrarse una profunda discusión sobre
las características de una investigación colaborativa y los dilemas
morales que puede plantear. Nosotros discutiremos cómo se ajusta este
estudio a lo que Feldman considera equitable collaborative research,
una vez explicada la metodología seguida.
\end{abstract}

${ }^{5}$ Este instrumento de análisis está diseñado para profesores de secundaria, por lo que algunos de sus indicadores necesitaron adaptación. Hemos hecho un uso flexible de éste, valorando los indicadores que no eran adecuados con una descripción de la situación que contemplábamos. Una tabla, dividida en cuatro tendencias (tradicional, tecnológica, espontaneísta e investigativa) resume este instrumento. Cada tendencia se describe en función de seis categorías (metodología, significado de la asignatura, concepción del aprendizaje, papel del alumno, papel del profesor y evaluación); estas categorías tienen varios descriptores, sumando un total de 35 descriptores por tendencia. Este instrumento es similar al presentado en Contreras, Climent y Carrillo (1999) para el análisis del papel que los profesores dan a la resolución de problemas en el aula.

${ }^{6} \mathrm{El}$ hecho de que el autor de las grabaciones fuera otra de las maestras o uno de los formadores era irrelevante; la elección se ha hecho en función de la mayor disponibilidad horaria del formador escogido.
${ }^{7}$ La diferencia reside en que, en la participación indirecta, los alumnos sólo intervienen en el diseño en la medida en que las reacciones de éstos son consideradas por el profesor a la hora de realizar el diseño futuro. Sin embargo, en la participación directa se les da opción a los propios alumnos a intervenir explícitamente en el diseño del proceso.

${ }^{8}$ Hemos usado en esta tabla y las que siguen las siglas y abreviaturas: CEAM (concepciones sobre la enseñanza y aprendizaje de las matemáticas), CmatEscolar (concepciones sobre la matemática escolar), CDC (conocimiento didáctico del contenido referido a la enseñanza de las matemáticas), Cde/sobreMat (conocimiento de y sobre matemáticas).

${ }^{9}$ Estos tres últimos se recogieron de manera indirecta al revisar, los investigadores, las notas de campo recogidas durante las sesiones.

${ }^{10}$ Las tres maestras (pero sobre todo Ana) habían manifestado sus mayores problemas en la enseñanza de los contenidos geométricos, respecto de los que se sentían con menor conocimiento profesional que en el tratamiento de otros contenidos matemáticos. Además, ha sido en el tratamiento de estos contenidos, coincidiendo con la segunda mitad del curso académico, cuando Ana ha decidido «poner manos a la obra» en la transformación de su práctica.

${ }^{11}$ Los niños poseen varios círculos recortados en papel.

${ }^{12}$ Cuando no se especifica nada, corresponde a las tres maestras.

${ }^{13}$ Del mismo modo que la preocupación de las maestras fue la que inició el proyecto y que la motivación de Ana hizo que se modificara y enriqueciera, el interés de éstas respecto a ciertos aspectos de su conocimiento profesional será el que determine en qué aspectos centrarán su desarrollo profesional.

${ }^{14}$ Éste es un aspecto que Feldman destaca como síntoma de que algunas de las investigaciones que analiza en su artículo no son totalmente colaborativas. Los informes de investigación suelen ser firmados sólo 
por el investigador y cuando aparecen los profesores suelen ocultarse sus nombres reales. Nos parece que estas cuestiones son discutibles. Los profesores pueden no estar interesados en esta tarea (su interés es su desarrollo profesional) y, por otro lado, pueden querer permanecer anónimos como profesionales sobre los que se aporta información abierta. De hecho, nosotros mismos, si realizáramos una investigación sobre nuestra práctica, probablemente preferiríamos no desvelarnos como los protagonistas de la práctica analizada.

${ }^{15} \mathrm{O}$ a los de los estudiantes para maestro, con lo que cabe pensar que el conocimiento de contenido necesita de algo más que la propia práctica para su desarrollo.

${ }^{16}$ Esto fue muy patente en el planteamiento de Ana de lo que era un polígono. Propuso a los niños una serie de figuras planas para que ellos sacaran la definición de polígono. Después, entre todos construyeron esa definición y Ana fue guiando hasta que llegaron a una definición que ella consideraba válida. Esta definición fue la siguiente: «Un polígono es una figura plana, cerrada y que tiene lados, vértices y ángulos.» Con esta definición pretenden excluir casos como figuras con algún lado curvo o figuras con más de un componente conexo, pero estas exclusiones no se justifican con la definición. Parece que ellos excluyen el primer caso porque, cuando piensan en lados, le están imponiendo que sean todos rectos. Por otra parte, el hecho de que posean lados, vértices y ángulos sería una consecuencia de que su contorno esté formado por una línea poligonal cerrada (hecho que excluye directamente las figuras con lados curvilíneos) y no una característica que los defina. Sin embargo, a pesar de que la definición consensuada pueda dar problemas, estos problemas no surgen, porque lo que todos los niños y la propia maestra están manejando no es la definición «verbal», sino el acuerdo al que han llegado respecto a las imágenes que consideran polígonos y las que no, la «definición mental» (definición del concepto frente a imagen del concepto [Tall y Vinner, 1981]).

${ }^{17}$ Este hecho puede explicar que, para ellas, la matemática que tiene sentido es la escolar, la única que conocen.

${ }^{18}$ Hecho generalizado en la enseñanza de la matemática en primaria en nuestro país, entre otras cosas porque los propios currículos y los centros de formación de profesores habían casi identificado la matemática de primaria con la aritmética y algo de álgebra. Es a partir de la reforma de 1990 cuando se restituye la importancia de los contenidos geométricos en esta etapa. Pero nos encontramos con generaciones de maestros en ejercicio y de estudiantes para maestro con escasos conocimientos básicos sobre geometría.

${ }^{19}$ Ella misma ha reconocido cómo nunca ha sido tan consciente de sus carencias de contenido matemático como en este proceso. Ahora hay muchas cuestiones de los alumnos, ideas que expresan, que, según sus palabras, «se le van de las manos».

${ }^{20}$ Preguntadas sobre este tema, Ana afirma que su conocimiento de contenido matemático se limita al de primaria, mientras que Lola afirma que, además de los contenidos de primaria, «domina» algunos propios de secundaria, por haber trabajado en esta etapa.

${ }^{21}$ Una excepción en este proceso ha sido el caso de Sara, en quien, debido a su menor implicación en el proyecto y grado de reflexión sobre su práctica, no se ha producido la toma de conciencia señalada. Esto nos ha llevado a no concluir el análisis del perfil de sus concepciones. 


\section{REFERENCIAS BIBLIOGRÁFICAS}

BALL, D.L. (1990a). The mathematical understanding that prospective teacher bring to teacher education. Elementary School Journal, 90, pp. 446-449.

BALL, D.L. (1990b). Prospective elementary and secondary teachers' understanding of division. Journal for Research in Mathematics Education, 21(2), pp. 132-144.

BALL, D.L. (1990c). «I haven't done these since high school»: prospective teachers' understnadings of mathematics. Proceedings of the $10^{\text {th }}$ PME-NA, pp. 268-274. Illinois.

BALL, D.L. (1996). Teacher learning and the mathematics reforms. What we think we know and what we need to learn. Phi Delta Kappan, 77(7), pp. 500-508.

BALL, D.L. y McDIAMIRD, G. (1988). Research on Teacher Learning: Studying how Teachers' Knowledge changes. Action in Teacher Education, 10(2), pp. 17-24.

BLANCO, L., MELLADO, V. y RUIZ, C. (1995). Conocimiento didáctico del contenido de ciencias y matemáticas y formación de profesores. Revista de Educación, pp. 307, 427-446.

BROMME, R. (1994). Beyond subject matter: A psychological topology of teachers' professional knowledge, en Biehler, R. et al. (eds.). Didactics of Mathematics as a Scientific Discipline. Dordrecht: Kluwer Academic Publisher.

CARRILlO, J. (1999). Conceptions and problem solving: a starting point and a tool for professional development, en Ellerton, N.F. (ed.). Mathematics Teacher Development. International Perspectives. West Perth, Australia: Meridian Press.

CARRILLO, J. y CORIAT, M. (1999). Madejas. Sugerencias sobre la formación del profesorado de secundaria. XXI Revista de Educación, 1, pp. 115-132.

CARRILLO, J., CORIAT, M. y OLIVEIRA, H. (1999). Teacher education and investigations into teacher's knowledge, en Krainer, K. y Goffree, F. (eds.). On Research in Mathematics Teacher Education. From a Study of Teaching Practices to Issues in Teacher Education. Osnabrück: University of Osnabrück. Capítulo 3, pp. 99-145.

CONTRERAS, L.C., CLIMENT, N. y CARRILLO, J. (1999). Teachers' beliefs on problem solving and mathematics education, en Krainer, K. y Goffree, F. (eds.). On Research in Mathematics Teacher Education. From a Study of Teaching Practices to Issues in Teacher Education. Osnabrück: University of Osnabrück. Capítulo 2, pp. 51-62.

COONEY, T. y KRAINER, K. (1996). Inservice Mathematics Teacher Education: The Importance of Listening, en Bishop,
A.J. et al. (eds.). International Handbook of Mathematics Education. The Netherlands: Kluwer Academic Publishers.

FELDMAN, A. (1993). Promoting equitable collaboration between university researchers and school teachers. Qualitative Studies in Education, 6(4), pp. 341-357.

IMBERNÓN, F. (2000). La formación del profesorado de secundaria. Entre la ausencia, el abandono y la desidia. Cuadernos de Pedagogía, 296, pp. 81-85.

JAWORSKI, B. (1998). Mathematics Teacher Research: Process, Practice and the Development of Teaching. Journal of Mathematics Teacher Education, 1(1), pp. 3-31.

KRAINER, K. (1998). Some Considerations on Problems and Perspectives of Inservice Mathematics Teacher Education, en Alsina, C. et al. (eds.). $8^{\text {th }}$ International Congress on Mathematics Education: Selected Lectures. Sevilla: SAEM Thales.

KRAINER, K. y GOFFREE, F. (1999). On research in mathematics teacher education. A short preview of the book, en Schwank, I. (ed.). European Research in Mathematics Education I. Proceedings of the First Conference of the European Society for Research in Mathematics Education, vol. 1. Osnabrück: Forschungsinstitut für Mathematikdidaktik.

PUIG, L. (1998). La didáctica de las matemáticas como tarea investigadora, en Puig, L. (ed.). Investigar y enseñar. Variedades de la educación matemática. Bogotá: Universidad de los Andes - Una Empresa Docente.

SCHÖN, D.A. (1992). La formación de profesionales reflexivos. Hacia un nuevo diseño de la enseñanza y el aprendizaje en las profesiones. Barcelona: Paidós-MEC. Ed. orig. (1987). Educating the reflective Practitioner. San Francisco: Jossey-Bass Publishers.

SHULMAN, L.S. (1986). Those who understand: knowledge growth in teaching. Educational Researcher, 15(2), pp. 4-14.

SIMON, M.A. (1994). Learning mathematics and learning to teach: learning cycles in mathematics teacher education. Educational Studies in Mathematics, 26(1), pp. 71-94.

STEINBRING, H. (1998). Elements of Epistemological Knowledge for Mathematics Teachers. Journal of Mathematics Teacher Education, 1(2), pp. 157-189.

TALL, D. y VINNER. S. (1981). Concept image and concept definition in mathematics, with particular reference to limits and continuity. Educational Studies in Mathematics, 12, pp. 151-169. 